\title{
Inequidades en la difusión y visibilidad del conocimiento: ¿por qué deberían importarnos? ${ }^{1}$
}

\author{
Inequities in the diffusion and visibility of knowledge: \\ why should we care? \\ Desigualdades na difusão e visibilidade do \\ conhecimento: por que devemos nos importar?
}

\author{
Clara Duarte Cuervo ${ }^{2}$ \\ Pamela Talero Cabrejo ${ }^{3}$ \\ Duarte Cuervo, C. y Talero Cabrejo, P. (2022). Inequidades en la difusión y visibilidad del \\ conocimiento: ¿ por qué deberían importarnos? Revista Ocupación Humana, 22(1), 3-11. \\ https://doi.org/10.25214/25907816.1372
}

En las dos últimas décadas, la difusión ${ }^{4}$ de conocimiento ha vivido un intenso proceso de mercantilización, centrado en la actividad de publicación. En este proceso, el artículo científico puede considerarse su principal mercancía, y las revistas han pasado a ser una condición que otorga o disminuye su valor (Collyer, 2016; Kiesslich et al., 2021; Salatino y López, 2021).

\footnotetext{
${ }^{1}$ Una versión abreviada de este texto fue presentada en el VI Seminário Nacional de Pesquisa em Terapia Ocupacional, de Brasil, el 2 de septiembre de 2021.

${ }^{2}$ Terapeuta ocupacional. Magíster en Salud Pública. Doctoranda en Terapia Ocupacional, Universidade Federal de São Carlos. Nemocón, Colombia. editorial@tocolombia.org iD) https://orcid.org/0000-0001-6901-7335

${ }^{3}$ Terapeuta ocupacional. Doctora en Terapia Ocupacional. Profesora asistente adjunta, Thomas Jefferson University. Filadelfia, Pensilvania, Estados Unidos de América. pamela.talero@jefferson.edu iD https://orcid.org/0000-0002-1057-9736

${ }^{4}$ De acuerdo con Estrada (2002), la difusión de conocimiento se refiere a los mecanismos utilizados para su diseminación al interior de las comunidades académicas y de investigadores, por ejemplo, los congresos, conferencias, revistas y artículos científicos. Se trata, entonces, de un concepto diferenciable del de divulgación (relacionada con estrategias dirigidas al público en general) y el de comunicación (atinente al intercambio de saberes y experiencias).
} 
Las revistas científicas se crearon como forma de comunicación y validación del conocimiento dentro de las comunidades científicas. Por eso, es común que fueran y sigan siendo editadas por asociaciones científicas o profesionales (como ocurre con buena parte de las revistas de Terapia Ocupacional alrededor del mundo) o por universidades. En ese contexto, la revisión por pares, además de ser una característica indispensable para que una revista científica sea considerada como tal, es central en esa validación que la misma comunidad hace del conocimiento que allí se construye (Kharasch et al., 2021). Esto es importante porque esa función de las comunidades científicas -de dar valor al conocimiento-, en ese proceso de mercantilización, es tomada por otros actores y, a su vez, cedida por tales comunidades. Trataremos de adentrarnos un poco en la manera como esto ocurre.

Figura 1. Circuitos dominantes del conocimiento

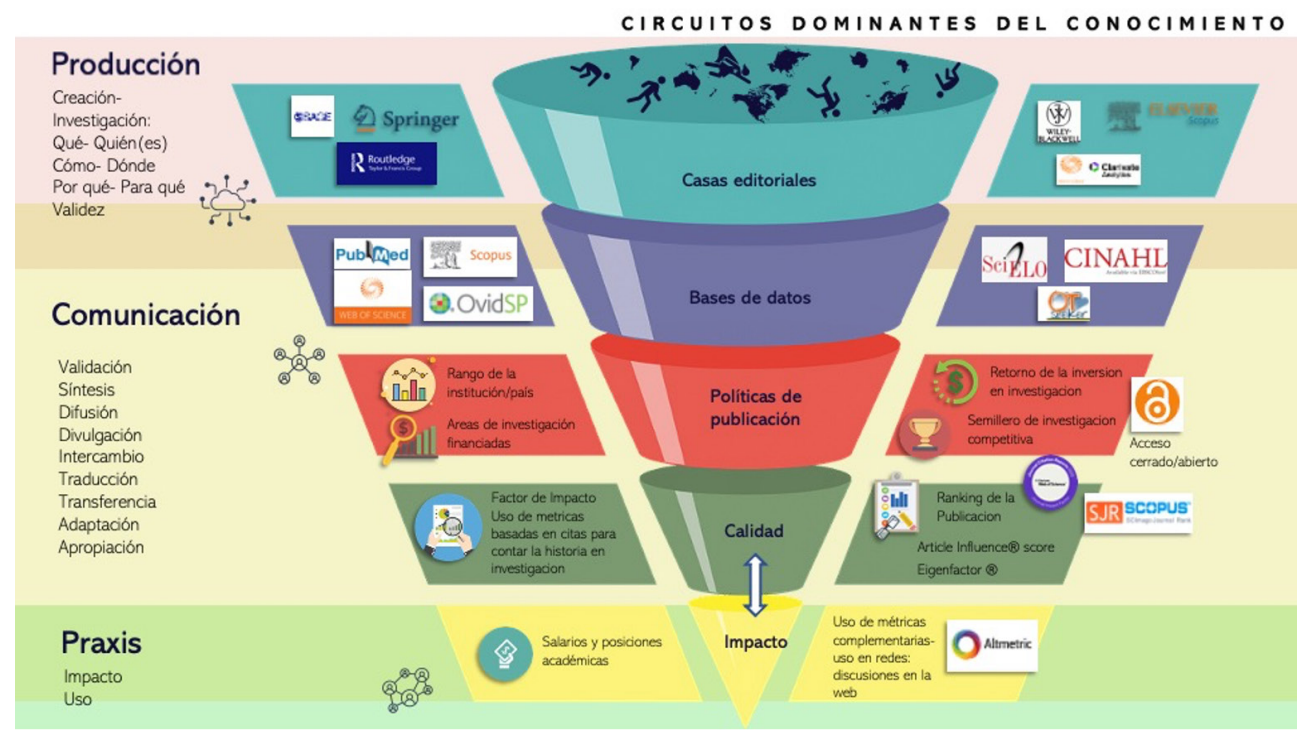

Fuente: elaboración propia.

Como se muestra en la figura 1, las bases de datos (o índices) son contenedores y organizadoras de revistas y artículos. Son útiles para que investigadores e investigadoras puedan lidiar con la cantidad de información científica que actualmente se produce y circula en el mundo. Existen bases temáticas, por campos de conocimiento o regionales, que abarcan ciertos nichos de esa producción ${ }^{5}$. Para que una revista puede ingresar en una base de datos científica, por lo general debe cumplir

${ }^{5}$ Por ejemplo Lilacs, en el caso de las ciencias de la salud en América Latina y el Caribe, una base de datos abierta y no comercial manejada por la Organización Panamericana de la Salud. 
con criterios técnicos relacionados con la calidad editorial. Hasta ahí, el sistema parece tener sentido y resultar útil.

No obstante, existen bases de datos con alcance internacional y multidisciplinario creadas por corporaciones editoriales multinacionales con fines de lucro. Entre estas bases están Web of Science (WoS) y Scopus. La primera es propiedad de la empresa Clarivate Analitycs, y la segunda pertenece a la editorial holandesa Elsevier. Estas empresas hacen parte de las seis editoriales dominantes en el mercado editorial mundial, las cuales manejan alrededor del $60 \%$ de las revistas incluidas en esos índices (Larivière et al., 2015; Shapiro, 2013).

Además de cumplir unos criterios de calidad editorial, para ser indexada en Web of Science o Scopus, una revista debe demostrar que es competitiva en ese entorno, esto es, que sus artículos son citados en las revistas ya incluidas en esa misma base de datos. Se trata, entonces, de un sistema hecho a la medida de las revistas que él mismo produce.

Como parte de su estrategia para consolidar el mercado del conocimiento, estas empresas han creado rankings para clasificar a las revistas (Clarivate/WoS tiene el Journal Citation Report- JCR y Elsevier/Scopus el Scimago Journal Rank-SJR). A través de estos, ofrecen a sus clientes (universidades, investigadores e investigadoras, estudiantes, comunidades académicas en general) un criterio a partir del cual valorarlas. A su vez, crean para sus productos en competencia (las revistas) un objeto de deseo: la posición en el ranking (cuartiles). Esta valoración -que pasa a ser asimilada con calidad del conocimiento- está basada en las citas que reciben los artículos y se traducen en indicadores, el más conocido de ellos, el factor de impacto: un indicador que tiene limitaciones bien documentadas como herramienta para la evaluación de la investigación (Kiesslich et al., 2021; Nature, 2005; Rozemblum et al., 2021; Salatino y López, 2021; Seglen, 1997; The Plos Medicine Editors, 2006; Vanclay, 2012).

Diversos estudios han mostrado que, si bien ha aumentado en los últimos años, las revistas latinoamericanas tienen una mínima participación en Web of Science y en Scopus (Repiso et al., 2019; Salatino, 2017; Salatino y López, 2021; Sobrido-Prieto et al., 2021). Esto se refiere tanto al número de revistas indexadas como al número de citas que reciben. Para 2017, solo el 2,3\% estaba indexado en Web of Science y el $8 \%$ en Scopus (Salatino, 2017). Buena parte de los países de Centroamérica y El Caribe no tienen ninguna participación en esas bases de datos (Salatino y López, 2021). Entre tanto, solo para tener una referencia, el estudio de Repiso et al., (2019) muestra que el 97\% de las revistas editadas por universidades, incluidas en el cuartil Q1 de Web of Science, correspondían a universidades británicas y de Estados Unidos.

Al mismo tiempo, bases de datos regionales abiertas y gratuitas como Latindex, Scielo y Redalyc, así como DOAJ, recogen buena parte de la literatura científica latinoamericana (Salatino y López, 2021). Estas fueron creadas para favorecer los diálogos, el intercambio y la colaboración regional, y promueven el acceso abierto 
al conocimiento. Aun así, son poco valoradas en los sistemas nacionales de evaluación de la ciencia y también son subestimadas en las revisiones de literatura.

Así, como en otros sectores en el mundo globalizado, la industria editorial ha logrado consolidar el mercado del conocimiento alcanzando una enorme influencia sobre todo el sistema: investigadores e investigadoras; publicaciones; grupos de investigación; universidades; sistemas de asignación de becas y recursos públicos y privados para investigación; sistemas de contratación, promoción y asignación salarial docente; políticas nacionales de ciencia, tecnología e innovación, entre otros. En todos estos niveles, la pertenencia y la ubicación en Web of Science y Scopus influyen de manera importante, profundizando brechas, inequidades y exclusiones; moldeando y transformando las prácticas científicas (Salatino y López, 2021), y generando distorsiones en el sistema de producción de conocimiento.

Una de estas distorsiones es que el valor atribuido a los artículos -y no el impacto social de las investigaciones- pasa a determinar el prestigio y el reconocimiento del investigador o investigadora. Quienes no alcanzan estos índices son presionados a aumentar la producción de cierto tipo de investigación y publicaciones, que no solo tienen el riesgo de ser apresuradas o hacer escasos aportes al conocimiento, sino de estar desconectadas de la realidad y de las necesidades locales (Kharasch et al., 2021). Esto crea a su vez una cultura de producción y consumo del conocimiento que le da más valor a aquello que se publica en revistas cientificas con un factor de impacto alto; revistas que, en su mayoría, son producidas en el norte global, en inglés y con la participación de empresas editoriales con fines de lucro.

Tal es el caso de Terapia Ocupacional. Como se muestra en la tabla 1, solo 11 revistas disciplinares estaban incluidas en el Scimago Journal Rank de Scopus para el año 2020, diez de ellas tienen su origen en el Norte global. Estados Unidos e Inglaterra controlan la producción con ocho. En este grupo hay solo una revista latinoamericana, los Cadernos Brasileiros de Terapia Ocupacional, que ocupa el puesto 11 y se ubica en el cuartil Q4 entre las revistas de alto impacto en la disciplina, con una marcada diferencia en los indicadores basados en citas respecto de aquellas que están en los primeros lugares. Todas estas revistas publican sus artículos en inglés, nueve de ellas exclusivamente en ese idioma. Nueve son manejadas total o parcialmente por las casas editoriales dominantes en el mercado: Wiley, SAGE o Taylor \& Francis.

¿Esto que implica? Por un lado, manda un mensaje claro de una hegemonía anglo-eurocéntrica del conocimiento en Terapia Ocupacional. Por otro, aunque nuestras y nuestros profesionales pueden publicar en esas revistas, tienen que ceñirse a reglas que no necesariamente rescatan el tipo de prácticas y formas de conocer como terapeutas ocupacionales de Latinoamérica. Adicionalmente, el papel y la autonomía de las comunidades profesionales o científicas para concertar, construir y definir la manera como valoran el conocimiento al interior de sí mismas queda en un tercer plano, dejando buena parte de estas decisiones en manos de las bases de datos y las editoriales. 
Tabla 1. Revistas de Terapia Ocupacional en Scimago Journal Rank (SJR) 2020

\begin{tabular}{|c|c|c|c|c|c|c|c|c|c|}
\hline Posición & País & Nombre & $\begin{array}{c}\text { Cuartil } \\
\text { SJR }\end{array}$ & $\begin{array}{c}\text { Índice } \\
\mathbf{H}\end{array}$ & $\begin{array}{c}\text { Total } \\
\text { citas } \\
(3 \\
\text { años) }\end{array}$ & Idioma(s) & Entidad a cargo & APC $^{6}$ & $\begin{array}{l}\text { Acceso } \\
\text { Abierto }\end{array}$ \\
\hline 1 & $\begin{array}{l}\text { Estados } \\
\text { Unidos }\end{array}$ & $\begin{array}{l}\text { American Journal of } \\
\text { Occupational } \\
\text { Therapy }\end{array}$ & Q1 & 82 & 650 & Inglés & $\begin{array}{l}\text { American } \\
\text { Occupational } \\
\text { Therapy } \\
\text { Association }\end{array}$ & No & No \\
\hline 2 & $\begin{array}{l}\text { Reino } \\
\text { Unido }\end{array}$ & $\begin{array}{l}\text { Australian } \\
\text { Occupational } \\
\text { Therapy Journal }\end{array}$ & Q1 & 44 & 340 & Inglés & $\begin{array}{l}\text { Wiley-Blackwell } \\
\text { Publishing Ltd/ } \\
\text { Occupational } \\
\text { Therapy Australia }\end{array}$ & Híbrido & Híbrido \\
\hline 3 & Canadá & $\begin{array}{l}\text { Canadian Journal of } \\
\text { Occupational } \\
\text { Therapy }\end{array}$ & Q1 & 55 & 180 & $\begin{array}{l}\text { Inglés/ } \\
\text { Francés }\end{array}$ & $\begin{array}{l}\text { SAGE Publications } \\
\text { Inc./ Canadian } \\
\text { Occupational } \\
\text { Therapy } \\
\text { Association }\end{array}$ & Sí & No \\
\hline 4 & $\begin{array}{l}\text { Reino } \\
\text { Unido }\end{array}$ & $\begin{array}{l}\text { Occupational } \\
\text { Therapy } \\
\text { International }\end{array}$ & Q2 & 37 & 133 & Inglés & Hindawi Limited & Sí & Sí \\
\hline 5 & $\begin{array}{l}\text { Estados } \\
\text { Unidos }\end{array}$ & $\begin{array}{l}\text { OTJR Occupation, } \\
\text { Participation and } \\
\text { Health }\end{array}$ & Q2 & 40 & 140 & Inglés & $\begin{array}{l}\text { SAGE Publications } \\
\text { Inc./The American } \\
\text { Occupational } \\
\text { Therapy } \\
\text { Foundation }\end{array}$ & Híbrido & Híbrido \\
\hline 6 & $\begin{array}{l}\text { Reino } \\
\text { Unido }\end{array}$ & $\begin{array}{l}\text { British Journal of } \\
\text { Occupational } \\
\text { Therapy }\end{array}$ & Q2 & 46 & 300 & Inglés & $\begin{array}{l}\text { SAGE Publications } \\
\text { Inc./ Royal } \\
\text { Collegue of } \\
\text { Occupational } \\
\text { Therapists }\end{array}$ & Híbrido & Híbrido \\
\hline 7 & $\begin{array}{l}\text { Reino } \\
\text { Unido }\end{array}$ & $\begin{array}{l}\text { Journal of } \\
\text { Occupational } \\
\text { Therapy, Schools, } \\
\text { and Early } \\
\text { Intervention }\end{array}$ & Q3 & 10 & 75 & Inglés & Taylor \& Francis & Sí & No \\
\hline 8 & $\begin{array}{l}\text { Hong } \\
\text { Kong }\end{array}$ & $\begin{array}{l}\text { Hong Kong Journal } \\
\text { of Occupational } \\
\text { Therapy }\end{array}$ & Q3 & 13 & 44 & Inglés & $\begin{array}{l}\text { SAGE } \\
\text { Publications/ } \\
\text { Hong Kong } \\
\text { Occupational } \\
\text { Therapy } \\
\text { Association }\end{array}$ & Sí & Sí \\
\hline 9 & $\begin{array}{l}\text { Estados } \\
\text { Unidos }\end{array}$ & $\begin{array}{l}\text { Occupational } \\
\text { Therapy in Health } \\
\text { Care }\end{array}$ & Q3 & 24 & 105 & Inglés & Taylor \& Francis & Sí & No \\
\hline 10 & $\begin{array}{l}\text { Reino } \\
\text { Unido }\end{array}$ & $\begin{array}{l}\text { Irish Journal of } \\
\text { Occupational } \\
\text { Therapy }\end{array}$ & Q3 & 2 & 12 & Inglés & $\begin{array}{l}\text { Emerald Group } \\
\text { Publishing Ltd./ } \\
\text { Association of } \\
\text { Occupational } \\
\text { Therapists of } \\
\text { Ireland }\end{array}$ & Híbrido & Híbrido \\
\hline 11 & Brasil & $\begin{array}{l}\text { Brazilian Journal of } \\
\text { Occupational } \\
\text { Therapy / Cadernos } \\
\text { Brasileiros de } \\
\text { Terapia } \\
\text { Ocupacional }\end{array}$ & Q4 & 4 & 65 & $\begin{array}{l}\text { Inglés/ } \\
\text { Portugués/ } \\
\text { Español }\end{array}$ & $\begin{array}{l}\text { Universidade } \\
\text { Federal de Sao } \\
\text { Carlos }\end{array}$ & Sí & Sí \\
\hline
\end{tabular}

Fuente: adaptado del reporte generado por Scimago Journal \& Country Rank (Scimago, 2021).

${ }^{6}$ La sigla APC corresponde a las iniciales en inglés de article processing charges (cargos por procesamiento de artículos). Esto es, los pagos que deben hacer los autores y autoras para publicar un artículo en una revista científica, incluyendo los que se cobran en sistemas híbridos para que el texto esté disponible para consulta en acceso abierto. 
Ahora bien, un elemento lingüístico fundamental en la disparidad en la difusión del conocimiento es que se privilegie el idioma inglés como lingua franca, más aún cuando las casas editoriales y los sistemas de evaluación del conocimiento incentivan la publicación en revistas con altos factores de impacto. Cuando la ciencia se comunica exclusivamente en inglés, arriesga su principal misión de informar al público (Céspedes, 2021; Federation of Finnish Learned Societies, 2019). Pero, fundamentalmente, erosiona las prácticas locales de elementos lingüísticos claves para entender y trabajar en el contexto social. Los datos sobre las revistas de Terapia Ocupacional en el Scimago Journal Rank, antes presentados, muestran que no hay incentivos suficientes para que las y los investigadores publiquen en su idioma nativo, ni para reconocer y estudiar el conocimiento que se produce en el Sur global. Esto limita la diseminación y el impacto de las revistas científicas latinoamericanas, pero también, la forma como pensamos, enseñamos e implementamos el conocimiento.

Otro elemento que no podemos pasar por alto es que los países de Latinoamérica, a excepción de Brasil y México, se encuentran en la periferia de las inversiones en ciencia, tecnología e innovación (Instituto de Estadística de la Unesco, 2021). Aun así, en un país como Colombia, cuyo presupuesto para educación y para investigación y desarrollo es limitado, algunas universidades invierten anualmente grandes montos en bases de datos (Bases de datos: ¿una pérdida de plata para las universidades?, 2017). A esto se suma el dinero que destinan -cuando esto ocurrea pagos por publicación y servicios de traducción para que sus investigadores e investigadoras puedan publicar en inglés en revistas indexadas en Web of Science y Scopus. Con frecuencia, una universidad -y un Estado, cuando se trata de universidades públicas- no solo financia la investigación, sino que termina pagando por publicarla y, luego, para poder acceder a ella.

Todo esto ha llevado a que los circuitos dominantes del conocimiento estén siendo cuestionados por actores del Sur y el Norte global, buscando afianzar modelos alternativos para la producción, la difusión y la apropiación social del conocimiento. Cobran allí sentido las iniciativas de ciencia abierta, así como las prácticas de cocreación y coproducción del conocimiento que propician encuentros de saberes dentro del contexto social e integran la praxis como elemento de origen, escritura y resultado del conocimiento. También, la creación de redes de cooperación en investigación Sur-Sur y Norte-Sur, y los proyectos de alcance global con aplicaciones locales.

Apuestas colectivas que buscan generar una masa crítica para la transformación de prácticas en los circuitos del conocimiento incluyen, entre otras, el Foro Latinoamericano sobre Evaluación Científica (FOLEC) (Consejo Latinoamericano de Ciencias Sociales [CLACSO], 2021); la Iniciativa Helsinki sobre Multilingüismo en la Comunicación Científica (Federation of Finnish Learned Societies et al., 2019); AmeliCA, Conocimiento Abierto sin fines de lucro propiedad de la academia (Becerril-García y Aguado-López, 2019), y la Declaración de San Francisco sobre la Evaluación de la Investigación [DORA] (DORA y Pardal-Peláez, 2018), de la cual 
la Revista Ocupación Humana es firmante. La recomendación principal de esta declaración es que no se utilicen métricas basadas en revistas -como el factor de impacto- como medida sustitutiva de la calidad de los artículos de investigación; para evaluar las contribuciones de un científico o científica, o en las decisiones de contratación, promoción o financiación.

La movilización del conocimiento implica no solo su transferencia, sino un proceso dinámico de reflexión y retroalimentación que valore las relaciones colectivas, el bienestar de otros y, por que no, un proceso social dinámico de resistencia, si se le mira desde un punto de vista de movilización social encaminada al diálogo, la negociación y el consenso (Cardinalli y Silva, 2021). Como Naidorf y Alonso (2018) lo explican, se trata de un proceso de tres tiempos que incluye la definición de agendas de investigación; el establecimiento de parámetros de evaluación de la investigación acordes con las necesidades nacionales y regionales, y el uso del conocimiento para la toma de decisiones de diferente tipo.

Entonces, vale la pena que nos preguntemos ¿quién y cómo se define el impacto del conocimiento? En Terapia Ocupacional, nuestro conocimiento no solo impacta nuestros fundamentos profesionales, sino que tiene efectos sobre la vida de alguien en algún lugar, especialmente de personas y grupos sociales que, por sus diferencias, son enajenadas a menos que contribuyan al sistema de producción y consumo. Por esto, el valor del conocimiento local es esencial.

Definir el impacto del conocimiento tiene que ver con quienes directamente se afectan o se benefician de lo que se investiga. Los procesos de conocimiento están ligados al contexto, por ello, deben ser considerados como un bien común público. En últimas, a quiénes leemos y citamos tiene repercusiones en cómo diseminamos los conocimientos de la región latinoamericana, pero también, en cómo pensamos, formamos y practicamos Terapia Ocupacional en nuestros países. Las disparidades en la producción, difusión y movilización del conocimiento, la desigualdad global como resultado de las desigualdades entre y dentro de los países, no solo tienen un efecto en nuestra presencia en las bases de datos y en las métricas por las cuales las y los investigadores reciben financiación y reconocimiento. Tienen un impacto directo en la salud, el bienestar y la vida de las personas y comunidades con las que cada terapeuta ocupacional trabaja en el cotidiano. 


\section{Referencias}

Bases de datos: ¿una pérdida de plata para las universidades? (2017, 9 de mayo). Universidades. Semana. https://www.semana. com/educacion/articulo/bases-de-datos-universitarias-presupuesto-para-investigacion-en-colombia/524572/

Becerril-García, A. y Aguado-López, E. (2019). Redalyc - AmeliCA. Un modelo de publicación sin fines de lucro para conservar la naturaleza académica y abierta de la comunicación científica. UNESCO, CLACSO y Redalyc.

Cardinalli, I. y Silva, C. R. (2021). Trajetórias singulares e plurais na produção de conhecimento de terapia ocupacional no Brasil. Cadernos Brasileiros de Terapia Ocupacional, 29. https://doi.org/10.1590/2526-8910. ctoAO2040

Céspedes, L. (2021). Revistas latino-americanas e línguas hegemônicas para publicação acadêmica no Scopus e Web of Science. Trabalhos em Linguística Aplicada, 60(1), 141-154. https://doi.org/10.159 o/010318138901311520201214

Collyer, F. M. (2016). Global patterns in the publishing of academic knowledge: Global North, global South. Current Sociology, 66(1), 56-73. https://doi. org/10.1177/0011392116680020

Consejo Latinoamericano de Ciencias Sociales [CLACSO] (2021). Foro Latinoamericano sobre Evaluación Científica (FOLEC). CLACSO. https://www.clacso.org/folec/

Declaration on Research Assessment [DORA] y Pardal-Peláez, B. (2018). Declaración de San Francisco sobre la evaluación de la investigación. Revista ORL, 9(4), 295-299. https://doi.org/10.14201/orl.17845
Estrada L. (2002). La divulgación de la ciencia. En J. Tonda, A. M. Sánchez y N. Chávez (coords.), Antología de la divulgación de la ciencia en México (pp. 138-151). Dirección General de Divulgación de la Ciencia, Universidad Nacional Autónoma de México.

Federation of Finnish Learned Societies; The Committee for Public Information; Publishing, The Finnish Association for Scholarly; Universities Norway; European Network for Research Evaluation in the Social Sciences and the Humanities (2019). HeIsinki initiative on multilingualism in scholarly communication. Figshare. Journal contribution. https://doi.org/10.6084/m9. figshare.7887059.v1

Instituto de Estadística de la Unesco [UIS] (2021). Gasto en I+D por país. Unesco. http://uis.unesco.org/apps/visualisations/ research-and-development-spendin$\mathrm{g} / \#$ !lang=es

Kharasch, E. D., Avram, M. J., Clark, J. D., Davidson, A. J., Houle, T. T., Levy, J. H., ... y Vutskits, L. (2021). Peer review matters: research quality and the public trust. Anesthesiology, 134(1), 1-6. https://doi. org/10.1097/ALN.0000000000003608

Kiesslich, T., Beyreis, M., Zimmermann, G. y Traweger, A. (2021). Citation inequality and the Journal Impact Factor: median, mean (does it) matter? Scientometrics, 126(2), 1249-1269. https://doi. org/10.1007/s11192-020-03812-y

Larivière, V., Haustein, S. y Mongeon, P. (2015). The oligopoly of academic publishers in the digital era. PloS one, 10(6), e0127502. https://doi.org/10.1371/journal.pone.0127502 
Naidorf, J. y Alonso, M. (2018). La movilización del conocimiento en tres tiempos. Revista Lusófona de Educação, 39(39). https://doi.org/10.24140/issn.1645-7250. $\underline{\text { rle39.06 }}$

Nature (2005). Not-so-deep impact. Nature, 435, 1003-1004. https://doi.org/10.1038/4351003b

Repiso, R., Orduña-Malea, E. y Aguaded, I. (2019). Revistas científicas editadas por universidades en Web of Science: características y contribución a la marca universidad. Profesional de la Información, 28(4), e280405. https://doi.org/10.3145/ epi.2019.jul.05

Rozemblum, C., Alperin, J. P. y Unzurrunzaga, C. (2021). Las limitaciones de Scopus como fuente de indicadores: buscando una visibilidad integral para revistas argentinas en ciencias sociales. e-Ciencias de la Información, 11(2). https://doi. org/10.15517/eci.v11i2.44300

Salatino, M. (2017). La estructura del espacio latinoamericano de revistas científicas [Tesis de doctorado, Universidad Nacional de Cuyo]. Biblioteca Digital UNCUYO. https://bdigital.uncu.edu.ar/10720

Salatino, M. y López, O. (2021). El fetichismo de la indexación. Una crítica latinoamericana a los regímenes de evaluación de la ciencia mundial. Revista Iberoamericana de Ciencia, Tecnología y Sociedad-CTS, 16(46).

Scimago (2021). Journal rankings. SJR Scimago Journal \& Country Rank. https:// www.scimagojr.com/journalrank.php?category $=3609 \&$ area $=3600 \&$ wos $=$ true $\&$ type $=\mathrm{j}$
Seglen, P.O. (1997). Why the impact factor of journals should not be used for evaluating research. The BMJ, (314), 498-502. https://doi.org/10.1136/bmj.314.7079.497

Shapiro, S. (2013). JSTOR, university presses, and the serials crisis. Journal of Electronic Resources Librarianship, 25(3), 240-242. https:// doi.org/10.1080/1941126X.2013.813319

Sobrido-Prieto, M., Talavera-Valverde, M. Á. y Souto-Gómez, A. I. (2021). Un estudio descriptivo de la presencia, visibilidad y calidad de las revistas de terapia ocupacional. Cadernos Brasileiros de Terapia Ocupacional, 29. https://doi. org/10.1590/2526-8910.ctoAO2205

The PLoS Medicine Editors (2006). The impact factor game. PLoS Medicine, 3(6), e291. https:// doi.org/10.1371/journal.pmed.0030291

Vanclay, J.K. (2012). Impact factor: outdated artefact or stepping-stone to journal certification. Scientometric, (92), 211-238. https://doi.org/10.1007/s11192-011-0561-0 